\title{
FRATURAS VERTEBRAIS ADJACENTES: CIFOPLASTIA VERSUS VERTEBROPLASTIA
}

\author{
ADJACENT VERTEBRAL FRACTURES: KYPHOPLASTY VERSUS VERTEBROPLASTY \\ FRACTURAS VERTEBRALES ADYACENTES: CIFOPLASTÍA VS VERTEBROPLASTÍA
}

luis Cláudio Schettino1, luis Eduardo Carelli Teixeira da Silva², Antônio Eulálio Pedrosa Araújo Júnior ${ }^{3}$,

Marcello Oliveira Barbosa ${ }^{4}$

RESUMO

Objetivo: Comparar a incidência de fraturas de vértebras adjacentes nos pacientes submetidos à vertebroplastia e cifoplastia. Métodos: Estudo retrospectivo baseado na análise de prontuários e exames radiográficos pré e pós-operatórios de janeiro de 2002 a janeiro de 2009. O seguimento mínimo foi de três meses. Os pacientes foram divididos em dois grupos distintos: Vertebroplastia (V) e Cifoplastia (C), de acordo com o procedimento realizado. Foram comparadas entre os dois grupos, a altura dos corpos vertebrais e a cifose angular antes e depois de cada procedimento e correlacionadas com a incidência de fraturas vertebrais adjacentes. A análise estatística foi feita pelos testes qui-quadrado, $t$ de Student e ANOVA de dois fatores seguida por teste post-hoc de Bonferroni. Resultados: Foram analisados os prontuários de 55 pacientes. Dez pacientes foram excluídos do estudo. Vinte e seis pacientes realizaram vertebroplastia e dezenove pacientes realizaram cifoplastia. Foi observada redução da cifose angular média nos pacientes do grupo C de 1,81 graus quando comparados aos pacientes do grupo V. Não houve diferença estatística entre os dois grupos quanto à altura média do corpo vertebral pré- e pós-operatória. Três pacientes do grupo $\mathrm{V}$ e dois pacientes do grupo $\mathrm{C}$ apresentaram fratura vertebral adjacente. Os dois pacientes com fraturas adjacentes do grupo $\mathrm{C}$ estavam em uso de corticosteroides. Os três pacientes do grupo $\mathrm{V}$ com fraturas adjacentes apresentaram extravasamento de cimento. Conclusão: Não houve diferença na incidência de fraturas vertebrais adjacentes entre os dois grupos.

Descritores: Vertebroplastia; Cifoplastia; Cifose; Fraturas da coluna vertebral; Estudos retrospectivos.

\begin{abstract}
Objective: To compare the incidence of adjacent vertebral fractures between vertebroplasty and kyphoplasty. Methods: We reviewed medical records and radiographs pre and postoperatively from January 2002 to January 2009. The minimal follow-up time was three months. The patients were divided in two distinct groups: vertebroplasty (V) and kyphoplasty (K) group, according to the procedure performed. The vertebral body height and the angular kyphosis before and after each procedure were compared between these two groups and correlated to the incidence of adjacent vertebral fractures. Results: We analyzed 55 medical records. Ten patients were excluded from the study. Twenty-six patients underwent vertebroplasty and nineteen patients underwent kyphoplasty. We found a reduction in the mean angular kyphosis of the kyphoplasty group of 1.81 degrees when compared to the vertebroplasty group. There was no statistical difference related to the mean vertebral body height preand postoperatively between the two groups. Three patients of the vertebroplasty group and two patients of the kyphoplasty group presented adjacent vertebral fracture. The two patients of the kyphoplasty group were corticoid-dependent. The three patients of the vertebroplasty group presented cement leakage. Conclusion: There was no difference in the adjacent vertebral fracture incidence between the two groups.
\end{abstract}

Keywords: Vertebroplasty; Kyphoplasty; Kyphosis; Spinal fractures, Retrospective studies; Adjacent vertebral fractures; Angular, cement leakage.

\section{RESUMEN}

Objetivo: Comparar la incidencia de fracturas de vértebras adyacentes en los pacientes sometidos a vertebroplastía y cifoplastía. Métodos: Estudio retrospectivo basado en el análisis de historias médicas y exámenes radiográficos pre y posoperatorios, de enero de 2002 a enero de 2009. El seguimiento mínimo fue de tres meses. Los pacientes fueron divididos en dos grupos diferentes: Vertebroplastía (V) y Cifoplastía (C), de acuerdo con el procedimiento realizado. Se comparó, entre los dos grupos, la altura de los cuerpos vertebrales y la cifosis angular, antes y después de cada procedimiento, y fueron correlacionadas con la incidencia de fracturas vertebrales adyacentes. El análisis estadístico fue hecho por las pruebas chi-cuadrado, $t$ de Student y ANOVA de 2 factores seguida por prueba post-hoc de Bonferroni. Resultados: Fueron analizadas las historias médicas de 55 pacientes. Diez pacientes fueron excluidos del estudio. Veintiséis pacientes realizaron vertebroplastía y diecinueve pacientes hicieron cifoplastía. Fue observada reducción de la cifosis angular promedio, en los pacientes del grupo C, de 1,81 grados cuando se compararon con los pacientes del grupo V. No hubo diferencia estadística entre los dos grupos cuanto a la altura promedio del cuerpo vertebral pre y posoperatoria. Tres pacientes del grupo $\mathrm{V}$ y dos pacientes del grupo $\mathrm{C}$ presentaron fractura vertebral adyacente. Los dos pacientes, con fracturas adyacentes del grupo C, estaban en uso de corticosteroides. Los tres pacientes del grupo V, con fracturas adyacentes, presentaron extravación de cemento. Conclusión: No hubo diferencia en la incidencia de fracturas vertebrales adyacentes entre los dos grupos.

Descriptores: Vertebroplastía; Cifoplastía; Cifosis; Fracturas de la columna vertebral; Estudios retrospectivos.

1. Médico Ortopedista, Chefe do Grupo de Doenças da Coluna do INTO - Rio de Janeiro, RJ, Brasil.

2. Médico Ortopedista, Chefe do Grupo de Trauma Raquimedular do INTO - Rio de Janeiro, RJ, Brasil.

3. Médico Ortopedista, Especialista em Cirurgia da Coluna e do Grupo de Doenças da Coluna do INTO - Rio de Janeiro, RJ, Brasil.

4. Médico Ortopedista, Especialista em Cirurgia da Coluna, Ex-estagiário do Grupo de Doenças da Coluna do INTO - Rio de Janeiro, RJ, Brasil.

Trabalho realizado no Instituto Nacional deTraumato-Ortopedia (INTO) - Rio de Janeiro, RJ. Brasil.

Correspondência: Marcello Oliveira Barbosa. Rua 36 sul lote 13 apto 503. Residencial Villa Pavanelli Il. Águas Claras. DF. Brasília. Brasil. CEP: 71.931-360- E-mail: mobarbos@ terra.com.br 


\section{INTRODUÇÃO}

As fraturas dos corpos vertebrais podem ser consequentes a neoplasias como o hemangioma, o mieloma múltiplo, as metástases ósseas e a osteoporose primaria e secundária ${ }^{1,2}$. A osteoporose é uma doença caracterizada pela diminuição da massa óssea e deterioração da microarquitetura do tecido ósseo (redução do trabeculado e afilamento cortical) com consequente aumento da fragilidade dos ossos e susceptibilidade a fraturas ${ }^{1,3}$. A principal manifestação clínica da osteoporose é a fratura patológica. Tem sido observado rápido aumento na incidência das fraturas vertebrais com o envelhecimento e incidência maior de fraturas vertebrais nas mulheres quando comparadas aos homens ${ }^{1}$. Tradicionalmente essas fraturas eram tratadas com o repouso no leito, colete gessado, fisioterapia e analgésicos. Contudo, cerca de um terço dos pacientes evoluem com dor persistente, refratária ao tratamento clínico habituall $^{1,2}$. Com o advento das técnicas minimamente invasivas de introdução de cimento cirúrgico (polimetilmetacrilato) por meio da vertebroplastia e da cifoplastia, tem havido aumento no número de pacientes tratados cirurgicamente ${ }^{4}$. A literatura é inconclusiva a respeito do risco aumentado de fraturas vertebrais adjacentes após estes procedimentos com taxas que variam amplamente para ambos os grupos, 8 a 52\% para a vertebroplastia e 3 a 39\% para a cifoplastia ${ }^{5,6}$. Fribourg et al. ${ }^{6}$ evidenciaram um aumento no numero de fraturas vertebrais adjacentes após a cifoplastia comparada a historia natural das fraturas vertebrais osteoporóticas nao tratadas. Estes autores demonstraram que $20 \%$ dos 40 pacientes estudados apresentaram fraturas vertebrais adjacentes, a maioria nos primeiros dois meses após o procedimento. Harrop et $\mathrm{al}^{7}$., estudando fraturas vertebrais adjacentes após cifoplastia e vertebroplastia afirmaram que variáveis como o alinhamento sagital global, o grau de osteoporose e a extensão do seu tratamento entre outras devem ser avaliadas antes de concluir que apenas o aumento da resistência da vértebra com a injeção do cimento seria responsável pelo desenvolvimento de fraturas futuras em níveis adjacentes.

O objetivo deste trabalho é comparar a incidência das fraturas das vértebras adjacentes nos pacientes submetidos aos procedimentos de vertebroplastia ou cifoplastia.

\section{MATERIAL E MÉTODOS}

O estudo é retrospectivo e foram pesquisados os pacientes com fraturas vertebrais secundárias à osteoporose, submetidos a vertebroplastia ou cifoplastia no período de janeiro de 2002 a janeiro de 2009 em um instituto terciário especializado em Ortopedia e em uma clínica privada. Os critérios de exclusão utilizados foram as fraturas vertebrais não-osteoporóticas, o seguimento inferior a três meses, o preenchimento incompleto do prontuário e a falta de exames. Foram analisados os prontuários e exames radiográficos pré e pós-operatórios de 55 pacientes. Foram incluídos no estudo 45 pacientes com análise de dados do prontuário e avaliação radiológica. Os pacientes foram separados em dois grupos distintos de acordo com o procedimento realizado: No Grupo "V" os pacientes submetidos a vertebroplastia e no grupo " $\mathrm{C}$ " os pacientes submetidos a cifoplastia. Foram analisadas a distribuição por gênero, a média de idade, a vértebra mais acometida, a cifose angular e a altura do corpo vertebral fraturado antes e depois dos procedimentos, e a incidência de fraturas vertebrais adjacentes em ambos os grupos. Foram comparadas entre os dois grupos de pacientes, a altura dos corpos vertebrais e a cifose angular após cada procedimento e correlacionadas com a incidência de fraturas vertebrais adjacentes. Todos os pacientes foram submetidos a exame de ressonância nuclear magnética antes dos procedimentos. Foi injetado cimento ósseo apenas nos corpos vertebrais fraturados que apresentavam edema ósseo detectado pela ressonância, indicando tratar-se de fratura recente. A punção da vértebra fraturada foi realizada introduzindo-se a agulha pelos pedículos até alcançar o corpo vertebral, sob anestesia local, usando-se xilocaína a 2\% com vasoconstrictor. A cifose angular pré e pós-operatória foi medida pelo método de Cobb em radiografias na incidência lateral em ortostase, traçando-se uma linha perpendicular à tangente ao platô inferior da vértebra fraturada e outra perpendicular tangente ao platô superior da vértebra de cima. A altura do corpo vertebral fraturado foi medida em milímetros, na incidência radiográfica em perfil antes e após a injeção de cimento. A análise estatística foi feita pelos testes qui-quadrado, $t$ de Student e ANOVA de 2 fatores seguida por teste post-hoc de Bonferroni.

\section{RESULTADOS}

Vinte e seis pacientes foram submetidos a vertebroplastia (Grupo V) e dezenove pacientes realizaram a cifoplastia (Grupo C). A média de idade do grupo $\mathrm{V}$ foi menor que a do grupo $\mathrm{C}$ e variou de 58 a 82 anos enquanto que no grupo $C$ essa variação foi de 50 a 88 anos. A proporção de homens e mulheres foi semelhante com prevalência de mulheres em ambos os grupos (Tabela 1). Foram detectadas 44 fraturas vertebrais osteoporóticas no grupo V. A vértebra mais acometida no grupo $V$ foi $L 1$ em 12 pacientes (35,3\%) seguida de T12 em 7 pacientes (20,58\%). Dez pacientes apresentavam fraturas em duas vértebras, dois pacientes apresentaram fratura em três e um paciente apresentou fratura em cinco vértebras simultaneamente neste grupo. Em relação ao grupo C, detectamos 24 fraturas vertebrais osteoporóticas por compressão. A vértebra mais acometida neste grupo também foi L1 em oito pacientes (42,10\%) seguida de T12 em cinco pacientes (Figura 1). Três pacientes apresentaram duas fraturas e um paciente apresentou três fraturas simultâneas no grupo C. Quanto à etiologia, 11 dos 19 pacientes do grupo $\mathrm{C}$ referiram que a fratura ocorreu por queda da própria altura. No grupo $\mathrm{V}, 16$ dos 26 pacientes também referiram que a fratura ocorreu por queda da própria altura. Dois pacientes faziam uso de corticoterapia de longa data no grupo $\mathrm{V}$ devido a artrite reumatóide e asma grave. No grupo $C$ três pacientes faziam uso crônico de corticóide devido a artrite reumatóide. O seguimento médio dos pacientes foi semelhante no dois grupos, variando de 3 a 30 meses e 11 dias no grupo $V$ e de 3 a 28 meses e 18 dias no grupo C (Tabela 1).

Tabela 1 - Características clínicas dos pacientes submetidos a vertebroplastia e cifoplastia.

\begin{tabular}{c|c|c}
\hline & $\begin{array}{c}\text { Grupo V } \\
\text { (Vertebroplastia) } \\
\mathbf{n = 2 6}\end{array}$ & $\begin{array}{c}\text { Grupo C } \\
\text { (Cifoplastia) } \\
\mathbf{n = 1 9}\end{array}$ \\
\hline Média de idade (anos) & $68 \pm 6,5$ & $74 \pm 7,4^{*}$ \\
\hline Relação H/M & $3 / 23$ & $2 / 17$ \\
\hline Vértebra mais acometida & $L 1(46,1 \%)$ & $\mathrm{L1}(42,1 \%)$ \\
\hline Etiologia mais frequente & $\begin{array}{c}\text { Queda da própria altura } \\
(61,5 \%)\end{array}$ & $\begin{array}{c}\text { Queda da própria altura } \\
(57,9 \%)\end{array}$ \\
\hline Uso de Corticoterapia & $2(7,7 \%)$ & $3(15,8 \%)$ \\
\hline Fratura em mais de uma vértebra & $12(46,1 \%)$ & $3(15,8 \%)^{*}$ \\
\hline$N^{0}$ de vértebras fraturadas & 44 & 24 \\
\hline Seguimento médio (em meses) & 8,27 & 7,47 \\
\hline
\end{tabular}

* médias estatisticamente diferentes ( $p<0,05$ com IC95\% e alfa 5\%).

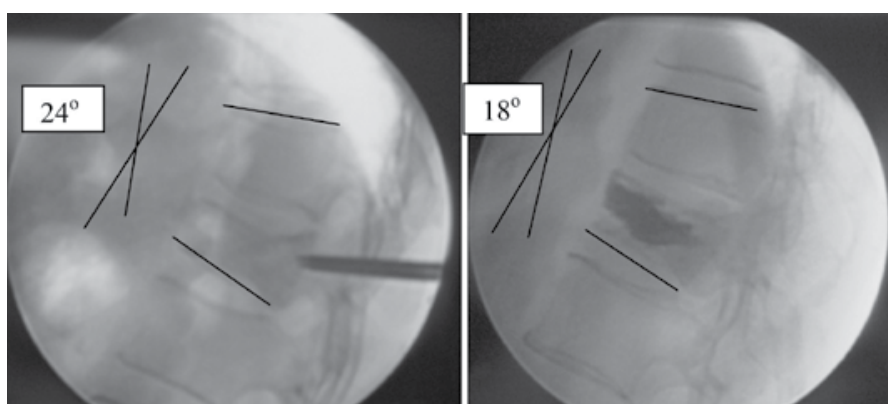

Figura 1. Fotos do intensificador de imagens em perfil da transição toracolombar mostrando elevação do platô superior de L1 após a cifoplastia com redução da cifose angular de 24 para 18 graus. 
A vertebroplastia foi realizada em 32 das 44 fraturas vertebrais detectadas nos 26 pacientes do grupo $V$ de acordo com os critérios já citados. Quatro pacientes foram submetidos a vertebroplastia em dois níveis e um paciente em três níveis. Os outros 21 pacientes realizaram o procedimento em um nível. A cifoplastia foi realizada em 22 das 24 fraturas vertebrais diagnosticadas no grupo C seguindo-se os mesmos critérios. Três pacientes realizaram a injeção de cimento em dois níves neste grupo e os demais 16 pacientes, em um nível. A cifose angular média pré-operatória das 32 vertebroplastias realizadas foi de 17,61 graus. A cifose angular média pós-operatória neste grupo foi de 16,68 graus. A redução média da cifose angular no grupo $\vee$ foi de 0,93 graus. A cifose angular média pré-operatória das 22 cifoplastias realizadas foi de 16,45 graus. No pós operatório, a média da cifose angular no grupo $C$ foi de 14,64 graus. A redução média da cifose angular neste grupo foi de 1,81 graus. A altura média do corpo vertebral fraturado antes da vertebroplastia era de 18,22mm. Após o procedimento a altura média subiu para 19,56mm. Antes da cifoplastia a altura média do corpo vertebral era de $17,04 \mathrm{~mm}$. Após a cifoplastia a altura média do corpo vertebral aumentou para 18,54mm (Tabela 2). A incidência de fraturas vertebrais adjacentes no grupo $V$ foi de três casos $(9,37 \%)$ enquanto que no grupo $\mathrm{C}$ foram detectadas duas fraturas adjacentes $(9,09 \%)$. Dentre as complicações mais frequentes observamos que o extravasamento de cimento foi prevalente em ambos os grupos. No grupo V, ocorreu em 15 (46,87\%) das 32 vertebroplastias realizadas. Em oito dos quinze casos, o extravasamento deu-se para o disco superior. No grupo $\mathrm{C}$ encontramos 8 casos $(36,36 \%)$ em 22 cifoplastias. Em cinco destes oito casos, também deu-se para o disco superior (Figura 2). Não houve extravasamento de cimento para o interior do canal medular em nenhum dos grupos.

Tabela 2 - Comparação da cifose angular média (CAM) e altura média do corpo (AMC) entres os dois grupos.

\begin{tabular}{c|c|c}
\hline & $\begin{array}{c}\text { Grupo V (Vertebroplastia) } \\
\mathbf{n = 2 6}\end{array}$ & $\begin{array}{c}\text { Grupo C (Cifoplastia) } \\
\mathbf{n = 1 9}\end{array}$ \\
\hline NNo de procedimentos $^{\circ}$ & 32 & 22 \\
\hline CAMpré-op. (em graus) & $17,61^{\circ}$ & $16,45^{\circ}$ \\
\hline CAMpós-op. (em graus) & $16,68^{\circ}$ & $14,64^{\circ}$ \\
\hline AMC pré-op. (em mm) & 18,22 & 17,04 \\
\hline AMC pós-op.(em mm) & 19,56 & 18,54 \\
\hline
\end{tabular}

Não houve diferença estatística entre os grupos.
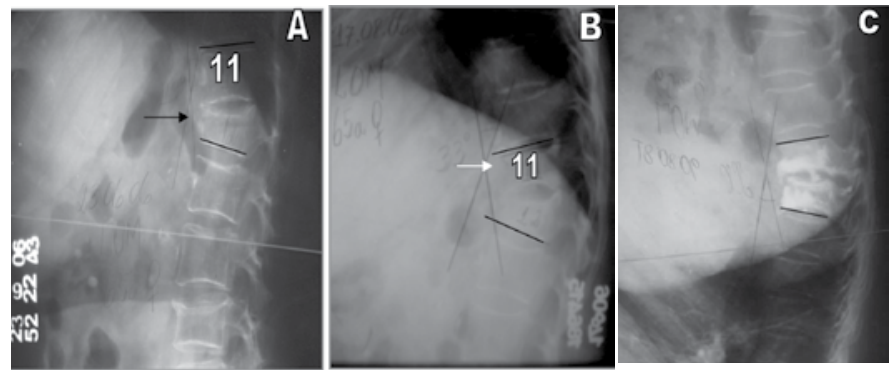

Figura 2. Radiografias em perfil de um mesmo paciente. (A) fratura de T12 (seta preta).(B) Fratura de T11 (seta branca) em menos de dois meses de seguimento, levando ao agravamento da cifose.(C) Leve redução da cifose angular após a vertebroplastia. Notar o extravasamento de cimento para o disco superior após a vertebroplastia de T12.

\section{DISCUSSÃO}

Foi possível observar que a injeção de cimento usando-se a técnica da vertebroplastia produziu uma pequena redução na cifose angular decorrente da fratura vertebral por compressão. A cifoplastia demonstrou redução maior da cifose angular (Figura 3) porém sem diferença estatística. Quanto à altura dos corpos vertebrais, após os procedimentos observamos que não houve redução signifi- cante dos platôs vertebrais superiores em nenhum dos grupos. Não observamos diferenças quanto à distribuição por gênero, presença de osteoporose secundária ao uso de corticoterapia e etiologia da fratura entre os grupos. Houve diferença quanto à média de idade que mostrou-se mais elevada no grupo $\mathrm{C}$ e quanto à fratura em mais de uma vértebra que foi maior no grupo V. A cifoplastia e a vertebroplastia tem sido associadas a um risco de fratura recorrente que varia de 2,4 a $23 \%$. A fratura pode ocorrer em qualquer vértebra. Contudo, dois terços delas ocorrem em níveis adjacentes dentro dos seis primeiros meses de pós-operatório ${ }^{7}$ (Figura 4). $\mathrm{Na}$ vertebroplastias, o extravasamento do cimento parece ter um importante papel na ocorrência de fraturas adjacentes. Por outro lado a influência do preenchimento do corpo vertebral fraturado com o polimetilmetacrilato na coluna vertebral não está claramente definida.
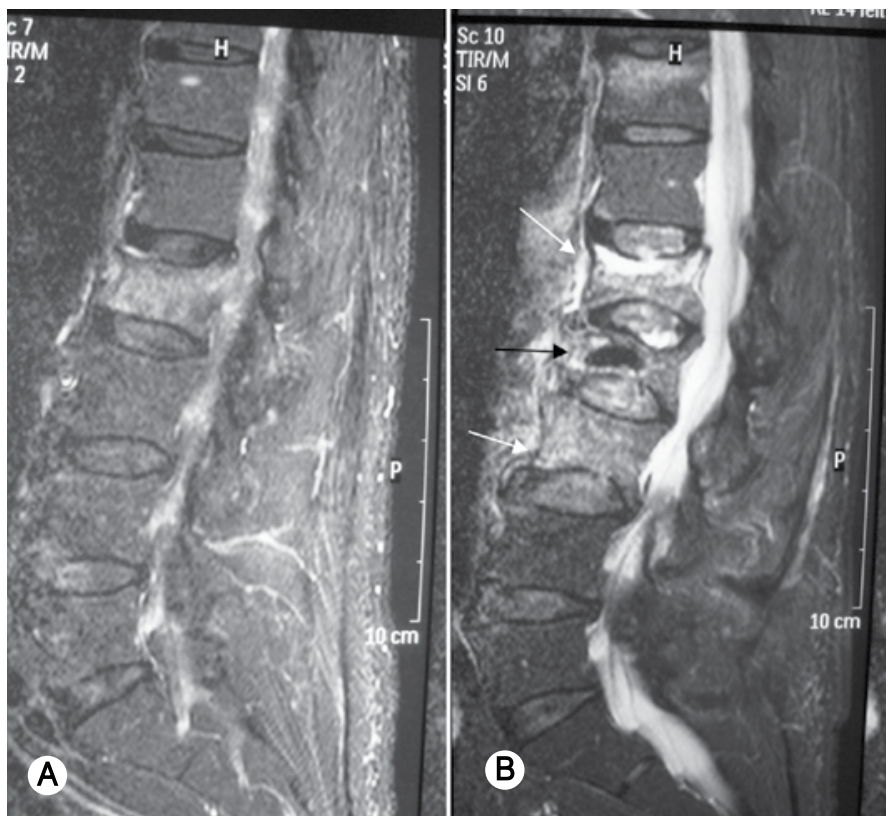

Figura 3. (A) Ressonância nuclear magnética da coluna lombar em cortes sagitais mostrando fratura do corpo de L2.(B) Fraturas adjacentes recentes dos corpos vertebrais de L1 e L3. Notar a presença de edema ósseo (setas brancas) destes corpos na imagem STIR após a cifoplastia de L2 (seta preta).

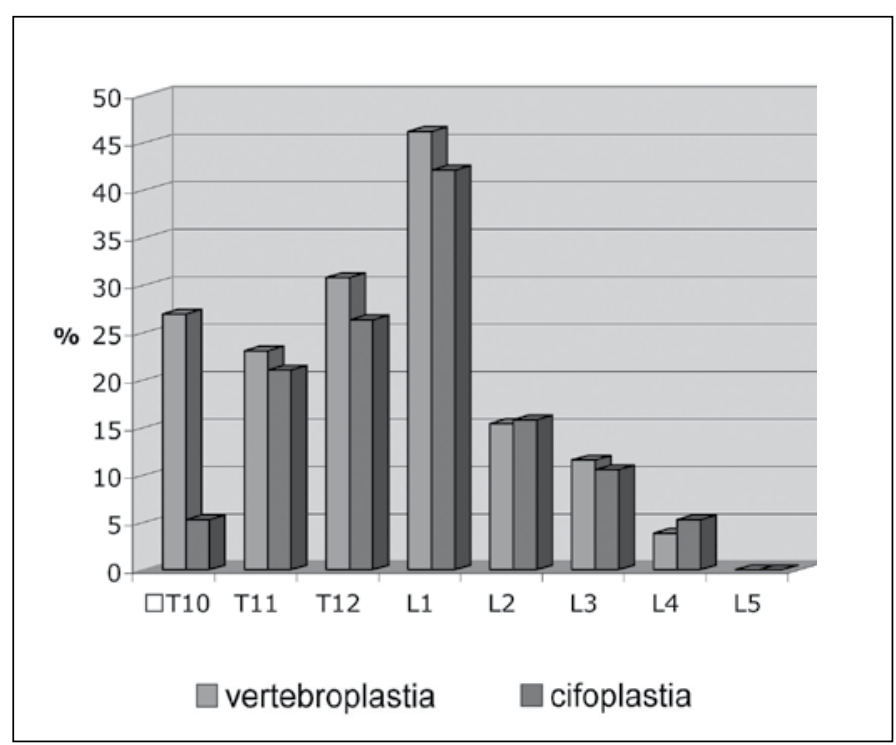

Figura 4. Distribuição do nível de fratura vertebral por compressão em pacientes submetidos a cifoplastia e vertebroplastia. 
Grados et al. ${ }^{8}$ examinaram esta questão em 25 pacientes submetidos a vertebroplastia após seguimento de 2 anos. Na sua casuística, 13 pacientes ou $52 \%$, apresentaram pelo menos uma fratura vertebral por compressão durante o período de seguimento ${ }^{8}$. Os autores postularam que este aumento do risco de fraturas estava relacionado da mudança na transferência de carga através da coluna vertebral como conseqüência do preenchimento de cimento no corpo da vértebra fraturada. Estes autores também notaram que 16 pacientes apresentam osteoporose primária e nove apresentavam osteoporose secundária, contudo não definiram esta associação com a incidência de fraturas subsequentes. Em nosso estudo não observamos a influência da corticoterapia na incidência das fraturas vertebrais adjacentes no grupo da vertebroplastia. Dos três pacientes que apresentaram fraturas adjacentes no grupo $V$, nenhum fazia uso crônico de corticosteróide. Todos os três apresentaram fratura do nível inferior. Um outro paciente deste grupo que apresentou fratura em nível remoto, também não fazia uso de corticosteróide. Já no grupo $\mathrm{C}$, dos dois pacientes que apresentaram fraturas vertebrais adjacentes ambos eram portadores de artrite reumatóide e faziam uso contínuo de corticosteróides. Os dois apresentaram fraturas nos níveis superior e inferior. A terceira paciente do grupo $\mathrm{C}$ que fazia uso de meticorten $5 \mathrm{mg}$ há 30 anos, portadora de artrite reumatóide, realizou cifoplastia em T12 e L2 e não apresentou fratura vertebral adjacente porém foi submetida a injeção profilática de cimento em L1. Harrop et al. ${ }^{7}$ encontraram uma incidência de $15 \%$ de fraturas vertebrais por compressão após a cifoplastia (34 em 225 procedimentos). A incidência de fraturas subsequentes ao procedimento no total de pacientes estudados foi de $22,6 \%$ (26 de 115 pacientes). Ao todo, estes resultados são favoravelmente comparados à história natural dos pacientes que apresentam mais de uma fratura vertebral por compressão cuja incidência publicada foi de $24 \%$ de acordo com Lindsay et al. ${ }^{9}$ Porém, quando estratificados em pacientes com osteoporose primária e osteoporose secundária observou-se uma incidência de fraturas vertebrais pós-cifoplastia de 11,25\% (9 em 80 pacientes) na osteoporose primária e de 48,6\% (17 em 35 pacientes) na osteoporose secundária em pacientes corticosteróide-dependentes. Portanto estes resultados inferem que a cifoplastia não aumenta o risco de fraturas em níveis remoto ou adjacente na osteoporose primária quando comparada ao histórico natural das fraturas vertebrais por compressão. Esses resultados também inferem que pacientes em uso crônico de corticosteróides, portadores de osteoporose secundária apresentam um risco aumentado para fraturas vertebrais por compressão após a cifoplastia (Figura 5) quando comparados aos pacientes com osteoporose primária, embora não haja nenhuma referência da história natural com a qual possamos comparar as incidências.

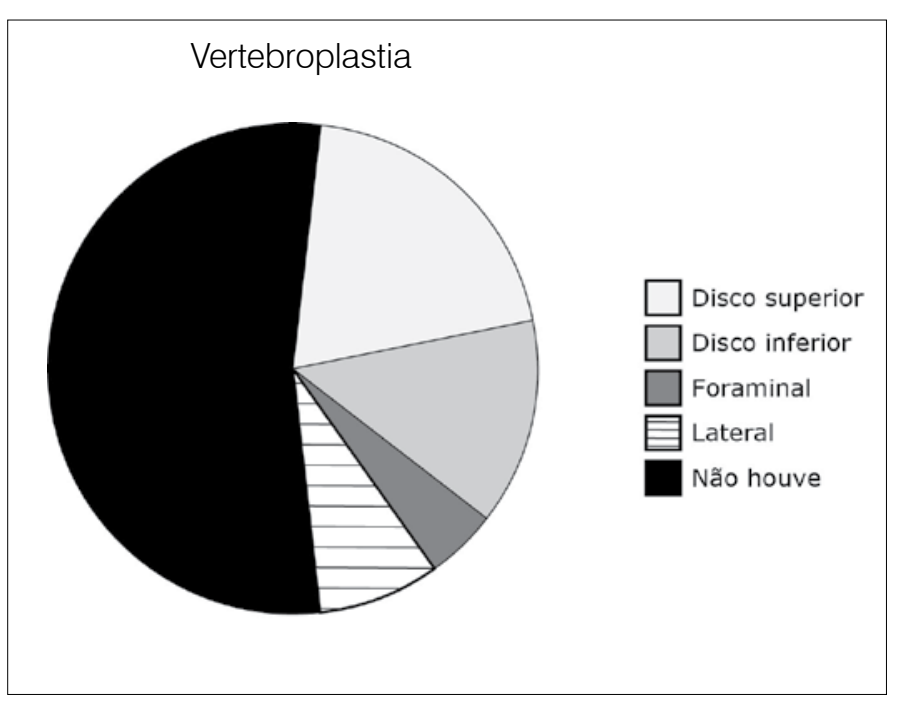

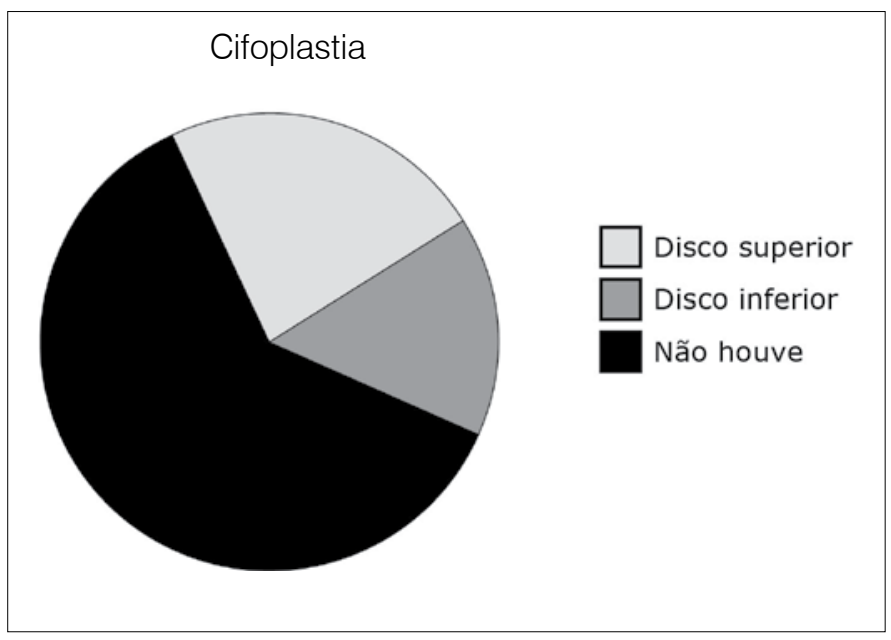

Figura 5. Comparação do extravasamento de cimento e a sua localização na vertebroplastia e na cifoplastia.

O extravasamento de cimento prevalente em ambos os grupos demonstrou-se presente nos três pacientes que apresentaram fraturas adjacentes após a vertebroplastia. Não observamos extravasamento de cimento nos dois pacientes do grupo $\mathrm{C}$ que apresentaram fraturas adjacentes. Baroud et al. ${ }^{10}$ desenvolveram modelos biomecânicos para estudar o efeito do preenchimento vertebral com cimento na carga exercida em vértebras adjacentes. A análise detalhada do modelo demonstrou que o cimento age como um sustentáculo, um pilar, dentro da vértebra tratada, reduzindo o abaulamento no interior das placas terminais. Como resultado deste efeito, a pressão no disco intervertebral adjacente aumentaria em torno de 19\%. Esses autores acreditam que essa mudança na carga adjacente é uma das razões que contribuem para a ocorrência de fraturas em níveis adjacentes ${ }^{10}$. Utilizando uma unidade funcional da coluna vertebral (UFCV) composta de dois corpos vertebrais de cadáver e o disco entre eles, Berlemann et al. ${ }^{11}$ demonstraram que a carga de colapso para a UFCV contendo um nível preenchido com cimento era significativamente menor (19\%) do que aquela para UFCV sem nível preenchido por cimento. Estes autores postularam que o enfraquecimento da UFCV poderia ser explicado por um efeito de elevação do "stress" no qual o aumento da rigidez da vértebra preenchida com cimento alteraria a transferência de carga para o nível adjacente não-cimentado. Lin et al. ${ }^{12}$ realizaram um estudo retrospectivo de pacientes tratados com vertebroplastia que apresentaram fraturas recorrentes que mostrou uma associação significante entre extravasamento de cimento para disco e essas fraturas. Em particular, esses autores demonstraram um risco aumentado de fraturas após vertebroplastias nos níveis adjacentes aos discos que apresentaram cimento extravasado. Estes achados estão de acordo com os efeitos biomecânicos conhecidos da vertebroplastia e credenciam a teoria que afirma que as fraturas de vértebras adjacentes devem estar relacionadas com o cimento injetado. É notável que outro estudo recente com um número maior de pacientes, desenvolvido por Syed et al. ${ }^{13}$, não tenha confirmado estes resultados. Havia, porém, neste estudo uma incidência aumentada de fraturas adjacentes em pacientes com extravasamento de cimento para o espaço discal que os autores não reconheceram (26 fraturas em 81 pacientes com extravasamento e 52 fraturas em 227 pacientes que não apresentaram extravasamento).

Existem várias explicações para a ocorrência de fraturas vertebrais adjacentes após o preenchimento do corpo vertebral com cimento. O nosso estudo não demonstrou diferença entre as duas técnicas quanto à incidência de fraturas adjacentes, quanto à altura do corpo vertebral e a redução da cifose angular. Porém a pequena casuística, o seguimento por período de tempo curto e o 
fato de tratar-se de estudo retrospectivo não permitem afirmar com exatidão se existe alguma vantagem relacionada a incidência de fraturas em vértebras adjacentes entre um ou outro procedimento. Três estudos de coorte recentes, publicados por Frankel et al. ${ }^{14}$ em 2007, Grohs et al. ${ }^{15}$ em 2005 e Pflugmacher et al. ${ }^{16}$ também em 2005 mostraram que novas fraturas foram um pouco mais frequentes após a cifoplastia quando comparada a vertebroplastia. Em um destes estudos, conduzido por Frankel et al. que publicou testes de significância, esta diferença foi estatisticamente significativa $(p<0,05)$. Porém tratou-se de estudo retrospectivo, com amostra inferior ao presente estudo (17 pacientes submetidos a cifoplastia e 19 pacientes submetidos a vertebroplastia) com seguimento mínimo de 6 meses $^{14}$. Os outros dois estudos, seguin- do na mesma linha deste, não mostraram diferença significante quanto à incidência de fraturas vertebrais adjacentes ${ }^{15,16}$. Até o momento, não existem estudos randomizados para comparar os dois procedimentos e portanto os dados disponíveis nos permitem apenas fazer uma análise indireta dos resultados. Estudos prospectivos randomizados são esperados para trazer maiores esclarecimentos quanto à incidência de complicações relativas a vertebroplastia e a cifoplastia.

\section{CONCLUSÃO}

Não foi observada diferença da incidência de fraturas vertebrais adjacentes após a realização de vertebroplastia ou cifoplastia nos pacientes desse estudo.

\section{REFERÊNCIAS}

1. Ledlie JT, Renfro MB. Kyphoplasty treatment of vertebral fractures: 2-year outcomes show sustained benefits. Spine (Phila Pa 1976). 2006:31(1):57-64

2. Cordeiro NGG, Cordeiro JORG, Vieira LAG. Vertebroplastia percutânea: uma efetiva técnica cirúrgica minimamente invasiva; Rev Bras Ortop. 2008;43(1/2):15-22.

3. Becker S, Garoscio M, Meissner J, Tuschel A, Ogon M. Is there an indication for prophylactic balloon kyphoplasty? A pilot study. Clin Orthop Relat Res. 2007;458:83-9.

4. Campbell PG, Harrop JS. Incidence of fracture in adjacent levels in patients treated with balloon kyphoplasty: a review of the literature. Curr Rev Musculoskelet Med. 2008:1(1):61-4

5. McCall T, Cole C, Dailey A. Vertebroplasty and kyphoplasty: a comparative review of efficacy and adverse events. Curr Rev Musculoskelet Med. 2008;1(1):17-23.

6. Fribourg D, Tang C, Sra P, Delamarter R, Bae H. Incidence of subsequent vertebral fracture after kyphoplasty. Spine (Phila Pa 1976). 2004;29(20):2270-6.

7. Harrop JS, Prpa B, Reinhardt MK, Lieberman I. Primary and secondary osteoporosis' incidence of subsequent vertebral compression fractures after kyphoplasty. Spine (Phila Pa 1976). 2004;29(19):2120-5.

8. Grados F, Depriester C, Cayrolle G, Hardy N, Deramond H, Fardellone P. Long-term observations of vertebral osteoporotic fratures treated by percutaneous vertebroplasty. Rheumatology (Oxford) 2000; 39:1410-1414.

9. Lindsay R, Silverman SL, Cooper C, Hanley DA, Barton I, Broy SB, et al. Risk of new vertebral fracture in the year following a fracture. JAMA. 2001;285(3):320-3.

10. Baroud G, Nemes J, Heini P, Steffen T. Load shift of the intervertebral disc after a vertebroplasty: a finite-element study. Eur Spine J. 2003;12(4):421-6.

11. Berlermann U, Ferguson SJ, Nolte LP, Heini PI, . Adjacent Vertebral failure after vertebroplasty. A biomechanical investigation. J Bone Joint Surg. Br. 2002; 84(5): 748-52.

12. Lin EP, Ekholm S, Hiwatashi A, Westesson PL. Vertebroplasty: cement leakage into the disc increases the risk of new fracture of adjacent vertebral body. AJNR Am J Neuroradiol. 2004;25(2):175-80.

13. Syed MI, Patel NA, Jan S, Harron MS, Morar K, Shaikh A. Intradiskal extravasation with low-volume cement filling in percutaneous vertebroplasty. AJNR Am J Neuroradiol. 2005;26(9):2397-401.

14. Frankel BM, Monroe T, Wang C. Percutaneous vertebral augmentation: an elevation in adjacent-level fracture risk in kyphoplasty as compared with vertebroplasty. Spine J. 2007;7(5):575-82.

15. Grohs JG, Matzner M, Trieb K, Krepler P. Minimal invasive stabilization of osteoporotic vertebral fractures: a prospective nonrandomized comparison of vertebroplasty and balloon kyphoplasty. J Spinal Disord Tech. 2005;18(3):238-42.

16. Pflugmacher R, Kandziora F, Schröder R, Schleicher P, Scholz M, Schnake K et al. [Vertebroplasty and kyphoplasty in osteoporotic fractures of vertebral bodies - a prospective 1-year follow-up analysis]. Rofo. 2005;177(12):1670-6. 